\title{
Lexical processing in a non-native language: Effects of language proficiency and learning strategy
}

\author{
HSUAN-CHIH CHEN \\ The Chinese University of Hong Kong, Shatin, New Territories, Hong Kong
}

\begin{abstract}
In three experiments, native Chinese speakers were asked to use their native and non-native languages to read and translate Chinese words and to name pictures. In Experiment 1, four groups of subjects with various degrees of proficiency in their second language, English, participated. In Experiments 2 and 3, subjects were first asked to learn a list of words in a new language, French, using either Chinese words or pictures as media; then they performed the reading, naming, and translation tasks. All subjects performed better in reading words than in naming pictures, when responding in Chinese. When the response was in the non-native language (English or French), high-learning subjects were equally efficient in translation and picture-naming tasks. Low-learning subjects, however, performed better in either the translation or the picture-naming task, depending on their learning strategies. These results are consistent with the idea that both proficiency in a non-native language and the strategy for acquiring the language are main determinants for the pattern of lexical processing in that language.
\end{abstract}

One important question in the study of bilingual or multilingual processing concerns how people process words in their non-native language. This question has been investigated by many researchers, using a variety of semantic tasks (see, e.g., Snodgrass, 1984, for a relevant review). These have included, for example, bilingual naming and translation (e.g., Chen \& Leung, 1989; Kroll \& Curley, 1987; Potter, So, Von Eckardt, \& Feldman, 1984), the semantic/sentence priming paradigm with a lexical decision task (e.g., Chen \& Ng, 1989; Jin \& Fischler, 1987; Kirsner, Smith, Lockhart, King, \& Jain, 1984; Kroll \& Borning, 1987; Schwanenflugel \& Rey, 1986), and the Stroop type of interference task (e.g., Biederman $\&$ Tsao, 1979; Chen \& Ho, 1986). The results of these studies with proficient, bilingual subjects are generally consistent with the concept-mediation hypothesis that the native and non-native languages of a proficient bilingual are operated independently, so that lexical items in the

This research was supported by a Faculty Research Grant from The Chinese University of Hong Kong. This article was prepared while the author was a Research Fellow at the Institute for Perception Research (IPO), and the recipient of a research fellowship from the Eindhoven University of Technology, the Netherlands. I am grateful to the principal, Chun Ping Ng, and the students of the Po Leung Kuk Siu HonSum Primary School for their kind assistance and participation; to YuenSum Leung, Siu-Yee Mak, Kin-Tong Chan, Siu-Yee Wong, and FungYing Siu for assistance in conducting the experiments; and to Mary Potter, Annette de Groot, James Chumbley, and an anonymous reviewer for helpful comments and suggestions on an earlier draft. Correspondence should be addressed to Hsuan-Chih Chen, Department of Psychology, The Chinese University of Hong Kong, Shatin, NT, Hong Kong. two languages are not directly associated but are connected through an amodal conceptual system (see Potter et al., 1984, for a detailed description of this hypothesis and related processing assumptions). However, whether beginning and proficient users of a non-native language process lexical items in similar ways in the new language has not been extensively investigated. The present study was designed to address this question and to test a relevant hypothesis proposed by Chen and Leung (1989).

A basic assumption in the present study is that there is a level of representation corresponding to different perceptual forms (e.g., pictures and different languages). These independent representations (e.g., lexicons and images), however, share a common representation at an abstract, conceptual level. This commonly accepted assumption has received support from studies on bilingual/multilingual processing and picture/word processing (for relevant results and discussion see, e.g., Bajo, 1988; Chen \& Ng, 1989; Potter, Kroll, Yachzel, Carpenter, \& Sherman, 1986; Potter et al., 1984; Schwanenflugel \& Rey, 1986; Snodgrass, 1984; Vanderwart, 1984). On the basis of this assumption, Figure 1 summarizes three processing models for bilingual subjects when the input stimuli are pictures of concrete objects and their corresponding words in a native language. These models will be referred to at various points in the present paper.

Potter et al. (1984) have previously found that both proficient Chinese-English and nonfluent English-French bilinguals had similar patterns of results in picturenaming, word-reading, and word-translating tasks: Both groups of subjects were much faster at word reading than 
at picture naming (the difference was above $250 \mathrm{msec}$ ) for responding in the first language (L1), whereas they were slightly slower at translating L1 words into the second language (L2) than at naming pictures in L2 (the difference was less than $60 \mathrm{msec}$ ). On the basis of these results, Potter et al. suggested that nonfluent and proficient bilinguals process lexical items in the two languages in similar ways (i.e., using the amodal, common concepts to mediate words in the two languages; see the conceptmediation pattern in Figure 1). These results thus seem to imply that language proficiency is not a major determinant for lexical processing in bilinguals.

However, Kroll and Borning (1987) have recently reported evidence to support the intermediate hypothesis that bilinguals originally use $\mathrm{L} 1$ words to mediate $\mathrm{L} 2$ words (i.e., the word-association pattern in Figure 1), but that, as their level of fluency in L2 increases, they switch to a stage of using amodal concepts to process $\mathrm{L} 2$ words (i.e., the concept-mediation pattern in Figure 1). In Kroll and Borning's study, English-Spanish bilinguals were given sentence fragments in English as primes, followed by target items in English or Spanish. The subjects had to judge whether the targets were legal words. Kroll and Borning found reliable priming effects for English targets for all subjects, whereas the pattern for Spanish targets was determined by the subjects' degrees of proficiency in Spanish (i.e., a significant priming effect was found only for more proficient subjects). Note that these results stand in interesting contrast with the findings of Potter et al. (1984). However, because there are many important differences between these two studies (e.g., in tasks: sentence priming and lexical decision vs. picture naming and reading/translating; in subjects: English-Spanish vs. Chinese-English and English-French), it is not clear whether these differences in method could have contributed independently or jointly to the various findings in the two studies.
More recently, Chen and Leung (1989) reported further evidence in support of the notion that beginning and proficient users of a non-native language process lexical items in the new language in different ways (see Kroll \& Curley, 1987, for similar findings). In Chen and Leung's study, native Chinese speakers with different degrees of proficiency in their non-native language (i.e., English for child beginners and adult proficient subjects or French for adult beginners) were asked to read words aloud, name pictures, and translate words. The results showed that all subjects were slower at picture naming than at word reading, when responding in Chinese. However, beginning and proficient subjects showed distinctively different patterns of response when responding in their non-native languages: Adult beginners were faster at translating than at picture naming, whereas child beginners were slower at translating than at picture naming; yet proficient subjects were equally efficient at translating and picture naming. Note that, although Chen and Leung adopted tasks and subjects similar to those used by Potter et al. (1984), the major patterns of results in these two studies are not consistent with each other.

To account for the different findings in earlier research, Chen and Leung (1989) proposed a modified, intermediate hypothesis. They suggested that proficient users of a non-native language could process words independently in both the native and the non-native languages to access their underlying, amodal concepts, whereas beginning users might need to use either words in the native language or pictorial representations, depending on the user's learning strategy, to mediate words in the new language. Thus, the word- or picture-association pattern (see Figure 1) may only be found at the beginning stage of acquiring a new language. Perhaps the nonfluent bilinguals in the Potter et al. (1984) study might have passed the beginning stage. Moreover, adult beginners of a nonnative language are more likely to use $\mathrm{L} 1$ words to ac-
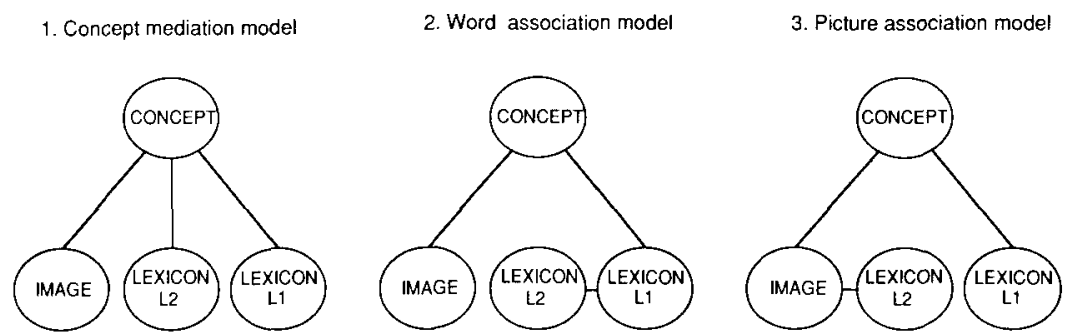

$$
\begin{array}{ll}
W 1>R 1: & (L 1) \\
P>R 1: & (1>C>L 1) \\
W 1>R 2: & (L 1>C>L 2) \\
P>R 2: & (I>C>L 2)
\end{array}
$$

(L1)
$(I->C+L 1)$
$(L 1>L 2)$
$(I>C>L 1>L 2)$

$$
\begin{aligned}
& (L 1) \\
& (1>C>L 1) \\
& (L 1>C>1>L 2) \\
& (1>L 2)
\end{aligned}
$$

Figure 1. An illustration of three models of bilingual processing of picture and word stimuli, with the processing steps in these models. $(P=$ picture, $C=$ concept, $I=$ image, $L 1=$ lexicon in Language $1, \mathrm{W1}=$ written word in Language 1 , and $\mathrm{R1}=$ response/spoken word in Language 1.) 
quire the new language, whereas child beginners are likely to use concrete learning media such as objects or pictures (see, e.g., Chen \& Leung, 1989). This is probably why the adult beginners in the studies of Chen and Leung, as well as those in Kroll and Borning (1987) and Kroll and Curley (1987), showed a lexical mediation pattern, whereas child beginners in Chen and Leung revealed a picture-mediation pattern.

The experiments reported in the present study were designed to test the intermediate hypothesis proposed by Chen and Leung (1989). This was done, in Experiment 1, by observing the development of patterns of lexical processing of Chinese-English bilinguals with different degrees of proficiency in their second language (English), whose experience in learning the new language ranged from 2 to 12 years. In Experiment 2, college students were instructed to acquire a set of words in an unfamiliar language while proficiency in the new language was experimentally manipulated. Finally, the possible effect of learning strategy was investigated in Experiment 3.

\section{EXPERIMENT 1}

Many previous researchers have demonstrated that beginning and proficient users of a second language show different patterns of results in processing words in the new language (e.g., Chen \& Leung, 1989; Kroll \& Borning, 1987; Kroll \& Curley, 1987). These results have been interpreted as supporting the idea that proficiency in L2 is a main determinant for patterns of lexical processing in bilinguals. A more rigorous way of testing this language-proficiency hypothesis would be to include different groups of bilingual subjects with varying degrees of proficiency in their second language. The languageproficiency hypothesis predicts that bilingual subjects' patterns of lexical processing should systematically change with their level of proficiency in L2. This prediction, however, was not tested in the studies mentioned above. Experiment 1 was thus conducted for such an end. To be consistent with the earlier research with which the present study was directly concerned (i.e., Chen \& Leung, 1989; Kroll \& Curley, 1987; Potter et al., 1984), three major tasks were used; they included picture naming in both $\mathrm{L} 1$ and $\mathrm{L} 2$, word reading in $\mathrm{L} 1$, and word translating from L1 to L2.

\section{Method}

Subjects. The participants in Experiment 1 were 96 native Cantonese speakers (Cantonese is a dialect of Chinese) from four grade levels (i.e., Grades 2, 4, and 6, as well as college), with 24 subjects at each level. There were 12 males and 12 females in each group. They were recruited from the Po Leung Kuk Siu Hon-Sum Primary School and from the Chinese University of Hong Kong. They had all learned Chinese (i.e., Cantonese) and English as their first and second languages, and they were Chinese-dominant in both reading and speaking, as reflected by their reading speeds for Chinese and English words. All the primary students had studied the English language at school for about 2, 4, or 6 years (i.e., for the second, fourth, and sixth graders, respectively). The undergradu- ate students had had over 12 years' training in English at various stages of school

Stimuli. The stimuli were 48 items of two types (line drawings of concrete objects and their corresponding names in Chinese). They were taken from the stimuli used by Chen and Leung (1989). All the Chinese items were originally found in textbooks from the first or second grade in primary school and were suitable for testing the primary students. All the pictures had familiar, unambiguous names in Cantonese and English.

The pictures and words were separately printed on $38 \times 25 \mathrm{~cm}$ cards, with 12 items on each card. Each card was constructed with three rows of 4 items of the same type of stimuli.

Procedure. The 48 items, divided into four cards of 12 stimuli each, were presented to all subjects. Each subject saw two cards in line-drawing form and another two in Chinese, and was required to respond in the native language (i.e., Cantonese) on half the trials and in the non-native language (i.e., English) on the other half. The order of administration of the cards (i.e., pictures or words) and the response language (native or non-native) were counterbalanced across subjects.

Note that the present procedure differed slightly from that used by Potter et al. (1984). The present procedure had been tested and used by Chen and Leung (1989), and had been shown to be equally as sensitive as the procedure of Potter et al. This simplified procedure was thus used in the reasonable confidence that it would provide a valid measure in the various conditions.

The subjects were tested individually. They were instructed to respond as accurately and as quickly as possible. They were also instructed not to point at the items while making their responses and to start from left to right and from top to bottom. Each trial began with instructions and a set of 10 practice items. Each test card was covered with a blank card and placed in front of the subject. The covered card was removed after a ready signal. The response time and the number of errors for each card were recorded.

\section{Results}

Response times. The mean response time per item was computed for each cell of the design. The data were subjected to an analysis of variance (ANOVA) with one between-subjects factor (grade level: $2,4,6$, or college) and two within-subjects factors (response language: native or non-native; and stimulus type: Chinese or picture). The mean response times for the various conditions are shown in Figure 2. The standard error of these mean values, as determined by the ANOVA, is 49.04. Post hoc comparisons were conducted using the protected $t$ test procedure (Fisher's least significant difference).

All main effects and the two-way and three-way interactions were significant at $p<.05$ : grade level $[F(3,92)=31.07]$, response language $[F(1,92)=$ 293.82], stimulus type $[F(1,92)=7.91]$, grade $\times$ language $[F(3,92)=13.75]$, grade $\times$ stimulus $[F(3,92)=$ 4.27], language $\times$ stimulus $[F(1,92)=132.43]$, and grade $\times$ language $\times$ stimulus $[F(3,92)=3.19]$. The most crucial finding was the significant three-way interaction (i.e., all other effects needed to be qualified by this).

These results revealed that when the subjects responded in Chinese, they were consistently faster with Chinese words than with pictures, at all grade levels $[t \mathrm{~s}(92)>$ $2.36, p s<.05]$. In fact, the magnitude of this word-overpicture superiority effect was relatively constant across different groups (i.e., 327, 353, 372, and $357 \mathrm{msec}$, from 


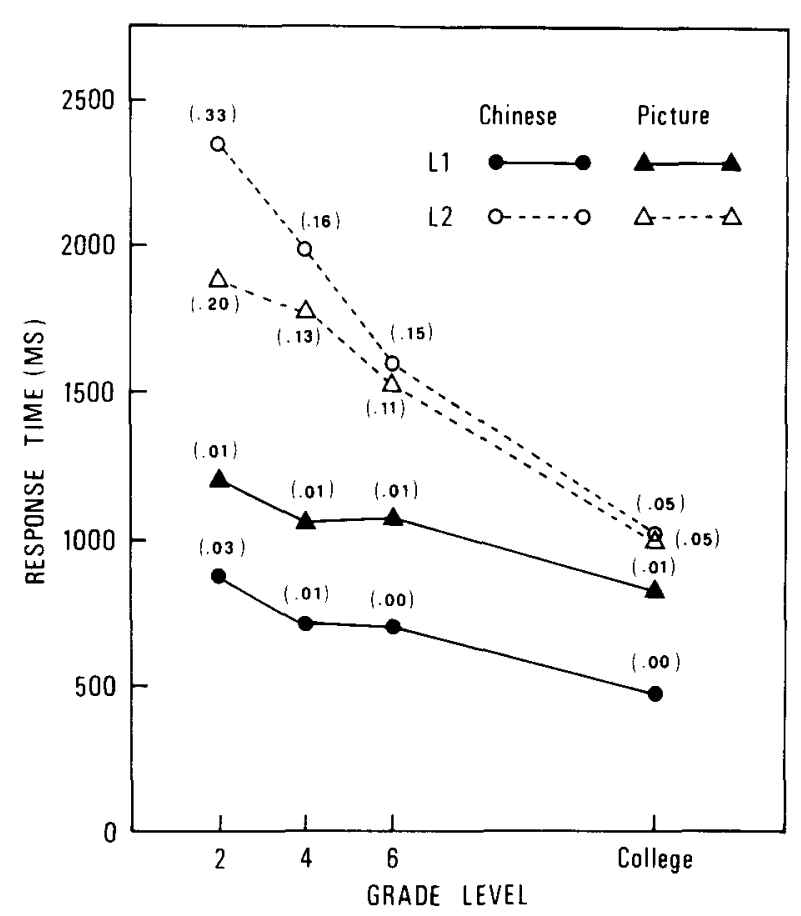

Figure 2. Mean response times (milliseconds per item) for Chinese words and pictured objects for two conditions of response language (Chinese/L1 and English/L2) plotted against grade level in Experiment 1 (error rates in parentheses).

Grade 2 to college, respectively). On the other hand, the patterns of results for the various groups of subjects were different when the responses were in English. Specifically, although the subjects were generally faster in naming pictures than in translating Chinese words into English, this difference systematically decreased as grade increased: The differences between naming and translating for different groups, from Grade 2 to college, were 471, 221, 132, and $25 \mathrm{msec}$, respectively. This difference was only significant for Grades 2 and 4 [ $t \mathrm{~s}(92)>3.19, p s<.05]$.

Moreover, when the responses were in English, response times decreased reliably as grade level increased (the means for different groups, from Grade 2 to college, were $2,112,1,875,1,528$, and $1,007 \mathrm{msec}$, respectively); except for the difference between Grades 2 and 4 , all other differences were significant $[t \mathrm{~s}(92)>2.70, p \mathrm{~s}<.05]$. When the responses were in Chinese, however, although a similar trend was found, it was not reliable (the means were $1,036,885,885$, and $653 \mathrm{msec}$, respectively); the only significant difference was found between Grade 2 and college subjects $[t(92)=3.04, p<.05]$. These results, as reflected by the significant grade $\times$ language interaction, indicate that the subjects' level of fluency in English (i.e., their second language) improved consistently from Grade 2 to college, whereas their fluency in Chinese (i.e., the native language) stabilized relatively early and had only a moderate improvement as educational level increased. Furthermore, subjects at all grade levels were faster when responding in Chinese than in English [all $t \mathrm{~s}(92)>2.80$, ps $<.05$ ], although this difference decreased as grade increased; the differences between the two response languages for the various groups, from Grade 2 to college, were $1,076,990,643$, and $355 \mathrm{msec}$, respectively.

Errors. Mean error rates were submitted to an ANOVA. These means are also shown in Figure 2, in parentheses. The standard error of these values, as determined by the ANOVA, is $1.8 \%$.

The analysis revealed that all main effects and the twoway interactions were significant at $p<.05$ : grade $[F(3,92)=15.29]$, language $[F(1,92)=147.99]$, stimulus $[F(1,92)=8.96]$, group $\times$ language $[F(3,92)=$ 14.79], group $\times$ stimulus $[F(3,92)=3.51]$, and language $X$ stimulus $[F(1,92)=8.89]$. The three-way interaction, however, was not significant $[F(3,92)=1.16]$. These results indicate that when the responses were in Chinese, the subjects made very few errors with both pictures and Chinese words ( $M \mathrm{~s}=1 \%$ and $1 \%$, respectively), whereas when responding in English, they generally had higher error rates with words than with pictures $[M \mathrm{~s}=17 \%$ and $12 \%, t(92)=2.08, p<.05]$. Furthermore, subjects from all grade levels made very few errors when responding in Chinese $(M s=1.9 \%, 1.2 \%, 0.5 \%$, and $0.5 \%$, from Grade 2 to college, respectively). On the other hand, the subjects made more errors when responding in English, but the error rates decreased as grade increased $(M \mathrm{~s}=26 \%, 14 \%, 13 \%$, and 5\%, respectively); except for the difference between Grades 4 and 6 , all other differences were significant $[t \mathrm{~s}(92)>2.60, p \mathrm{~s}<.05]$. Moreover, the second graders made more errors with Chinese words than with pictures $[M \mathrm{~s}=18 \%$ and $11 \%$, $t(92)=2.96, p<.05$ ], whereas no difference was found between words and pictures for all other groups.

Adjusted response times. Note that the response time data obtained in the present experiment included both erroneous and accurate responses. One might reasonably ask whether this could have contributed to the response time results mentioned above. Chen and Leung (1989) had previously used both the single-trial procedure, in which correct and error responses were experimentally separated, and the present procedure, and they had demonstrated that results obtained under different procedures were highly compatible. Nevertheless, to partial out possible influences of errors on reaction time data, the response times were submitted to an analysis of covariance (ANCOVA), with error rate as a single covariate. The idea was to assess effects of manipulated variables on response times while possible influences due to the subjects' having made various amounts of errors in different conditions were statistically controlled. The adjusted average response times are reported in Table 1 .

All the significant effects obtained in the previous ANOVA were also obtained in the ANCOVA: grade level $[F(3,91)=14.73$; the covariate term, $F(1,91)=24.25$, $p<.05]$, response language $[F(1,91)=75.78]$, grade $\times$ language $[F(3,91)=6.76$; the covariate term, $F(1,91)=9.05, p<.05]$, stimulus type $[F(1,91)=$ $8.81]$, grade $\times$ stimulus $[F(3,91)=3.24$; the covariate 
Table 1

Adjusted Average Reaction Times (in milliseconds) for Chinese Words and Pictures for Four Groups of Subjects Using Either Chinese (L1) or English (L2) as the Response Language in Experiment 1

\begin{tabular}{lccccc}
\hline & \multicolumn{4}{c}{ Response Language } \\
\cline { 2 - 3 } \cline { 5 - 6 } \multicolumn{1}{c}{ Group } & Chinese & Picture & & Chinese & Picture \\
\hline Second graders & 913 & 1,254 & 2,154 & 1,780 \\
Fourth graders & 762 & 1,112 & & 1,925 & 1,729 \\
Sixth graders & 758 & 1,127 & & 1,536 & 1,440 \\
College students & 533 & 879 & 1,041 & 1,022 \\
\hline
\end{tabular}

term, $F(1,91)=0.92, p>.34]$, language $\times$ stimulus $[F(1,91)=112.81]$, and grade $\times$ language $\times$ stimulus $[F(3,91)=2.71$; the covariate term, $F(1,91)=2.18$, $p>.05]$. In fact, the overall patterns of these results were highly consistent with those from the previous analyses of the original response times.

\section{Discussion}

In Experiment 1, a constant effect of word-over-picture superiority was found for subjects of all levels (i.e., about $350 \mathrm{msec}$ ) when the response language was L1. This result is consistent with findings in prior research (e.g., Chen \& Leung, 1989; Kroll \& Curley, 1987; Potter et al., 1984). Furthermore, for the subjects with very little knowledge of L2 (i.e., second and fourth graders), picture naming in L2 was significantly more efficient than was translating L1 words into L2 (the differences were about 471 and $221 \mathrm{msec}$, respectively). This result is also in line with previous findings of Chen and Leung.

The present results for child beginners of a second language are consistent with the findings of Chen and Leung (1989), indicating that child learners of a new language (i.e., child beginners) are probably using pictorial representations but not $\mathrm{L} 1$ words as media to process corresponding words in the new language. Alternatively, the child beginners might still have used common concepts rather than pictorial representations to mediate and produce $\mathbf{L} 2$ responses, but the picture-to-concept link was better developed than the L1-to-concept link, because the young beginners might not have been very proficient in reading $\mathrm{L} 1$ words. This hypothesis, however, has been previously tested and rejected by Chen and Leung (1989), who found that for child beginners, the relative times to understand an L1 written word and a picture were not statistically different.

Another possible interpretation of the picture-over-word superiority effect for the child beginners (i.e., second and fourth graders) is that the translation task might have caused Stroop-like interference between the L1 word and the corresponding $\mathrm{L} 2$ response. The young children might have experienced greater interference than college subjects in the translating task, probably because the young subjects were still in the process of learning written $\mathrm{L} 1$ words, and they tended to decode L1 words into their spoken forms. This interpretation, however, is inconsis- tent with findings from previous studies. For example, with bilingual subjects similar to those in the present study (i.e., Chinese-English bilinguals of different grades), Chen and Ho (1986) demonstrated that younger subjects did not necessarily show greater color-naming interference than did older subjects such as college students, when the stimulus and the response were in different languages.

The most crucial finding in the present study is that the magnitude of the picture-over-word superiority effect gradually decreased as the subjects' proficiency in L2 increased. In fact, although the sixth graders still tended to be faster at picture naming than at translating (the difference was about $132 \mathrm{msec}$ ), this difference was no longer reliable. For subjects with more than 12 years training in L2 (i.e., the college subjects), picture naming and translating were actually equally efficient (the difference was only about $25 \mathrm{msec}$ ).

Note that, in the present study, subjects of different groups not only had different degrees of proficiency in $\mathrm{L} 2$, but also varied in other aspects (i.e., their fluency in L1 and the amount of errors they made when responding in L2). One might reasonably ask whether these factors (i.e., language proficiency in L1 and number of errors in L2) could have contributed significantly to the present findings. This is not likely, except in the case of the youngest group of subjects, because the present results indicate that the subjects' level of fluency in $\mathrm{L} 1 \mathrm{had}$ stabilized at early stages of education (see Chen \& Ho, 1986, for similar findings), as reflected by the fact that when responding in L1, no significant difference was found among fourth graders, sixth graders, and college subjects. This was particularly true when we compared the results of fourth and sixth graders. The subjects in these two groups not only were equally efficient in Ll, but also had very similar patterns of errors. However, their results in L2 were clearly different (i.e., a reliable effect of pictureover-word advantage was found for the fourth graders, whereas this effect was no longer reliable for the sixth graders). Furthermore, when the possible influences of errors on reaction times were partialed out statistically, the pattern of results remained unchanged. Taken together, these results indicated that when responses were in the new language, the patterns of results changed systematically with subjects' proficiency in the new language.

\section{EXPERIMENT 2}

Experiment 2 was designed to test further the possible effects of proficiency in a non-native language on lexical processing. The optimal way to do this without confounding by subject factors would be to include subjects with similar individual and social characteristics (e.g., age, intelligence, educational history) but with varied experiences of the new language. Thus, in Experiment 2, two groups of college students, rather than subjects of different grades, were included. Both groups received the same kind but different amounts of training in learning words in an unfamiliar language (French). The French words 
were paired with corresponding $\mathrm{Ll}$ words as study stimuli. This word-learning strategy was used, because it has been reported to be a common strategy for acquiring words in a new language among adult subjects (Chen \& Leung, 1989; Kroll \& Borning, 1987; Kroll \& Curley, 1987). At the end of the learning phase, the subjects were asked to perform the reading, naming, and translating tasks used in Potter et al. (1984). If the amount of learning is a main determinant in patterns of lexical processing in the new language, one would expect to find that the difference between translating Chinese into French and picture naming in French would be greater in the low-learning group than in the high-learning group.

\section{Method}

Subjects. The participants in this research were 40 Cantonesespeaking undergraduates, who were recruited from the introductory psychology subject pool at The Chinese University of Hong Kong. These subjects had learned Chinese as their first language and had no knowledge of French. None of these subjects had participated in Experiment 1.

Stimuli. Twenty line drawings and corresponding words in both Chinese and French were used as stimuli. The stimuli formed 20 French-Chinese pairs. Each pair was printed on a $15 \times 10 \mathrm{~cm}$ white card for presentation in a Gerbrands $\mathrm{T}-4 \mathrm{~A}$ four-field tachistoscope. In addition, all the line drawings and the Chinese items were also printed individually on $15 \times 10 \mathrm{~cm}$ white cards.

Procedure. The subjects were randomly divided into two groups and run individually. The experiment included a study phase and a test phase. In the study phase, one group of subjects (the lowlearning group) received 20 French-Chinese pairs repeated three times, and another group of subjects (the high-learning group) received the same French-Chinese pairs repeated nine times. The stimuli were presented at a rate of $5 \mathrm{sec} /$ pair, using the tachistoscope. The subjects were instructed to concentrate on each stimulus pair as it was presented, in preparation for a later memory test.
During the presentation of each stimulus pair, a female experimenter read the presented French word to the subject once.

After all the stimulus pairs were presented, a test phase started. The test phase included four different tasks (naming pictures in Chinese and in French, reading Chinese items in Chinese, and translating Chinese items into French) like those in Experiment 1. However, the administration of the tasks in Experiment 2 was slightly modified, so that all the test items, including 10 Chinese items and 10 line drawings, were randomly and individually presented at a rate of $3 \mathrm{sec} /$ item, using the tachistoscope. The subjects' responses to each item, including both response time and accuracy, were recorded individually. This was done with a timer and a voice-detection key connected to the tachistoscope. The timer was activated as soon as a stimulus item appeared, and it was stopped by the voice key. The voice key was activated when the subject made his or her oral response. The subjects were instructed to make a response only when the item was still in view and to respond as quickly and as accurately as possible. The four tasks were blocked. The order of tasks and the assignment of 20 stimuli to four tasks (i.e., five test items were presented in each task) were counterbalanced across subjects.

To prevent direct study-test transfer, the Chinese items were printed in different forms (i.e., the standard and the scribe forms) in the study and test phases. Half the subjects in each group saw one form of the Chinese items in the study phase, and the other half of the subjects saw another form.

\section{Results and Discussion}

Response times. The mean response times for correct responses were computed for each subject in all cells of the design. The data were analyzed using an ANOVA with one between-subjects factor (amount of learning: high or low); and two within-subjects factors (response language: Chinese or French; and stimulus type: Chinese item or picture). The mean results are shown in Figure 3. The standard error of these values, as determined by the ANOVA, is 62.5.

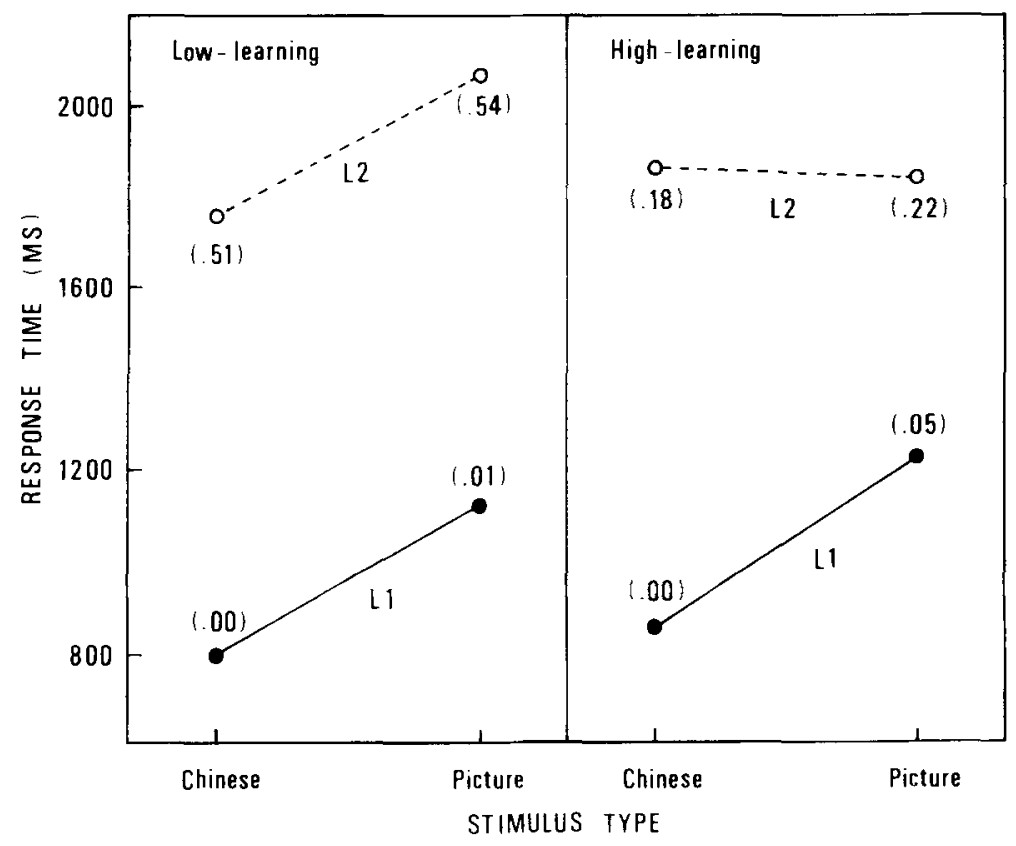

Figure 3. Mean response times in naming or translating Chinese words (L1) and pictured objects as a function of amount of learning in Experiment 2 (error rates in parentheses). 
The analysis revealed the following significant main effects and interactions: response language $[F(1,38)=$ 336.54], stimulus $[F(1,38)=48.06]$, learning $\times$ stimulus $[F(1,38)=4.19]$, response $\times$ stimulus $[F(1,38)=$ $10.56]$, learning $\times$ response $\times$ stimulus $[F(1,38)=8.62$; all $p s<.05]$. These results could be understood by examining the most critical finding, the three-way interaction (see Figure 3). Clearly, when subjects were requested to respond in the new language, the patterns of results for the two subject groups were different: Chinese items produced significantly faster responses than did pictures for the low-learning group [Ms $=1,758$ and $2,063 \mathrm{msec}$, respectively; $t(38)=4.87, p<.01]$, whereas no similar difference was found for the high-learning group (Ms $=1,861$ and 1,839 msec, respectively). These results are thus consistent with the idea that amount of learning is the main determinant for different patterns of lexical processing.

Furthermore, because the high-learning group received more training in L2 than the low-learning group did, one might reasonably ask why their overall performance in L2 did not significantly differ from that for the lowlearning group (i.e., $M \mathrm{~s}=1,850$ and 1,910 $\mathrm{msec}$ for the high- and low-learning groups, respectively). This is probably due to the fact that the high-learning group was generally slower than the low-learning group (i.e., $M \mathrm{~s}=1,045$ and 955 msec for the high- and low-learning groups, when the response language was $\mathrm{L} 1$ ).

Moreover, as demonstrated in Experiment 1 and in many other previous studies (e.g., Potter \& Faulconer, 1975; Potter et al., 1984), responses in the subjects' native language, Chinese, were faster with Chinese items than with pictures for both groups of the subjects [both $t \mathrm{~s}(38)>3.4, \mathrm{ps}<.01]$.

Errors. Mean error rates were computed and subjected to an ANOVA. The mean error rates for the various conditions are shown in Figure 3. The standard error of these mean values, as determined by the ANOVA, was $2.3 \%$. The analysis revealed four significant effects or interactions: learning $[F(1,38)=26.79]$, response language $[F(1,38)=151.76]$, response $\times$ learning $[F(1,38)=$ $37.40]$, and stimulus $[F(1,38)=4.7$; all $p \mathrm{~s}<.05]$. These results indicate that low-learning subjects made many more errors $(M=52.5 \%)$ than did high-learning subjects $(M=20 \%)$ when using French as the response language $[t(38)=8.12, p<.01]$, whereas no significant difference was found between the two groups when the response language was Chinese $(M \mathrm{~s}=0.5 \%$ and $2 \%)$. These findings, in conjunction with the response time results, clearly suggest that the high-learning subjects were more efficient than the low-learning subjects in using the newly acquired French words, but that the two groups were equally accurate in using their native language.

\section{EXPERIMENT 3}

The results of Experiment 1, in conjunction with findings from previous studies (e.g., Chen \& Leung, 1989;
Kroll \& Curley, 1987; Potter et al., 1984), indicated that child and adult beginners showed different patterns of lexical processing for words in the new language. It was hypothesized that this difference was due to the fact that the child and adult beginners might have used different learning strategies to acquire the new language. Experiment 3 was designed to investigate whether learning strategy could affect the strategy for processing words in the new language at the beginning stage of language learning. Two groups of college subjects were instructed to learn words in an unfamiliar language (i.e., French) according to different learning strategies. These new words were paired with corresponding pictures as study stimuli for one group of subjects (i.e., the picture-learning group), and they were paired with corresponding L1 words for another group of subjects (the word-learning group). If learning strategy was indeed a determinant of the pattern of lexical processing for beginning learners of a new language, one would expect to find that at the beginning stage of learning, the word-learning group should be more efficient in translating $\mathrm{L} 1$ words into the new language than in naming pictures in the new language, whereas the picture-learning group should be faster in the picturenaming than in the translating task.

\section{Method}

Subjects. Forty Cantonese-speaking undergraduates at The Chinese University of Hong Kong volunteered to participate. They had learned Chinese as their first language and had no knowledge of French. None of them had participated in Experiment 1 or 2.

Stimuli. The stimuli were 20 line drawings and their corresponding words in both Chinese and French, similar to those used in Experiment 2. However, in the present experiment, these stimuli formed both French-Chinese and French-picture pairs.

Procedure. The subjects were randomly divided into two groups and run individually in three continuous sessions, which lasted for about $30 \mathrm{~min}$. Each session consisted of a study phase and a test phase. In the study phase, one group of subjects (i.e., the picturelearning group) received 20 French-picture pairs, and another group of subjects (the word-learning group) received 20 French-Chinese pairs, presented at a rate of $5 \mathrm{sec} /$ pair using the tachistoscope. After all the stimulus pairs had been presented, a test phase started.

In each test phase, five test items were presented in each test condition. For a given subject, the order of different test conditions was constant in three test phases, and so were the test items in each condition (but the order of different test items in each condition was randomized in each test phase). In other words, each subject was tested with the same test form for a given French word on each of the test trials. The general procedure in the learning and test phases was similar to that used in Experiment 2.

The three sessions were basically the same, except that the list of stimulus pairs was presented once in the study phase of the first session, twice in the second session, and three times in the third session (the display order of the pairs was randomized in each trial). This procedure was adopted to ensure that subjects could achieve a higher degree of proficiency in response to the French words in each session relative to its previous session.

\section{Results and Discussion}

Response times. The mean response times for correct responses were computed for each subject in all cells of the design. Since all subjects performed very poorly when 
responding in French in the first session, these data were not used for statistical analysis. The remaining data were analyzed with an ANOVA with one between-subjects factor (learning condition: word or picture) and three withinsubjects factors (session: two or three; response language: Chinese or French; and stimulus type: Chinese item or picture). The mean results are shown in Figure 4. The standard error of these values, as determined by the ANOVA, is 47.3.

The analysis revealed the following significant main effects and interactions: session $[F(1,38)=19.02]$, response language $[F(1,38)=612.89]$, stimulus $[F(1,38)=36.35]$, stimulus $\times$ group $[F(1,38)=21.06]$, response $\times$ stimulus $[F(1,38)=48.68]$, session $\times$ stimulus $\times$ group $[F(1,38)=18.76]$, response $\times$ stimulus $\times$ group $[F(1,38)=9.09]$, and session $\times$ stimulus $\times$ response $\times$ group $[F(1,38)=7.28$; all $p$ s $<.02]$. These results can be understood by examining the four-way interaction (see Figure 4). Clearly, when subjects were requested to respond in their newly acquired language, French, the patterns of results for picture- and wordlearning groups were completely opposite at the early stages of learning: Chinese items produced faster responses than did pictures for the word-learning group ( $M \mathrm{~s}=1,659$ and 1,991 msec, respectively), whereas an opposite pattern, of Chinese producing slower responses than pictures did ( $M \mathrm{~s}=1,893$ and 1,590 $\mathrm{msec}$, respectively), was found for the picture-learning group [both $t \mathrm{~s}(38)>4.5, p \mathrm{~s}<.01]$. These results are thus consistent with the notion that subjects' learning strategies at the early stage of L2 learning is a main determinant for the patterns of lexical processing.

However, when subjects had a certain amount of training in French (i.e., Session 3), no significant difference was found between translating Chinese items into French and naming pictures in French for both the word-learning group ( $M \mathrm{~s}=1,688$ and $1,645 \mathrm{msec}$, respectively) and the picture-learning group $(M \mathrm{~s}=1,558$ and $1,520 \mathrm{msec}$, respectively). Note that since a repeated-test design was adopted in Experiment 3, the subjects had had multiple exposures to the Chinese and picture versions of the corresponding French words in different test phases. One might reasonably ask whether the factor of repeated testing could have contributed to the result of the third session (i.e., that subjects responded equally efficiently to both translating and naming in French tasks). This is not very likely, however, because a similar pattern of results was also found in the high-learning condition in Experiment 2 , where a single-test rather than a repeated-test design was adopted. The present results, in conjunction with the findings of Experiment 2, thus indicate that the amount of learning is a key factor in determining the pattern of lexical processing in a non-native language.

Furthermore, as demonstrated in Experiments 1 and 2 and in many other relevant studies (e.g., Potter \& Faulconer, 1975; Potter et al., 1984), responses in the subjects' native language were faster with Chinese items than with pictures, for both groups of the subjects [ts(38) > 3.4, ps <.01].

Errors. Mean error rates were computed and subjected to an ANOVA. The mean error rates for various conditions are shown in Figure 4. The standard error of these mean values, as determined by the ANOVA, was $2.9 \%$. The analysis revealed three significant effects or interactions: session $[F(1,38)=74.57]$, response language $[F(1,38)=163.66]$, and response $\times$ session $[F(1,38)=$ 49.88; all $p s<.001]$. These results indicate that the subjects made more errors in Session $2(M=49 \%)$ than in Session $3(M=28 \%)$ when using French as the response language $[t(38)=7.12, p<.01]$, whereas no signifi-

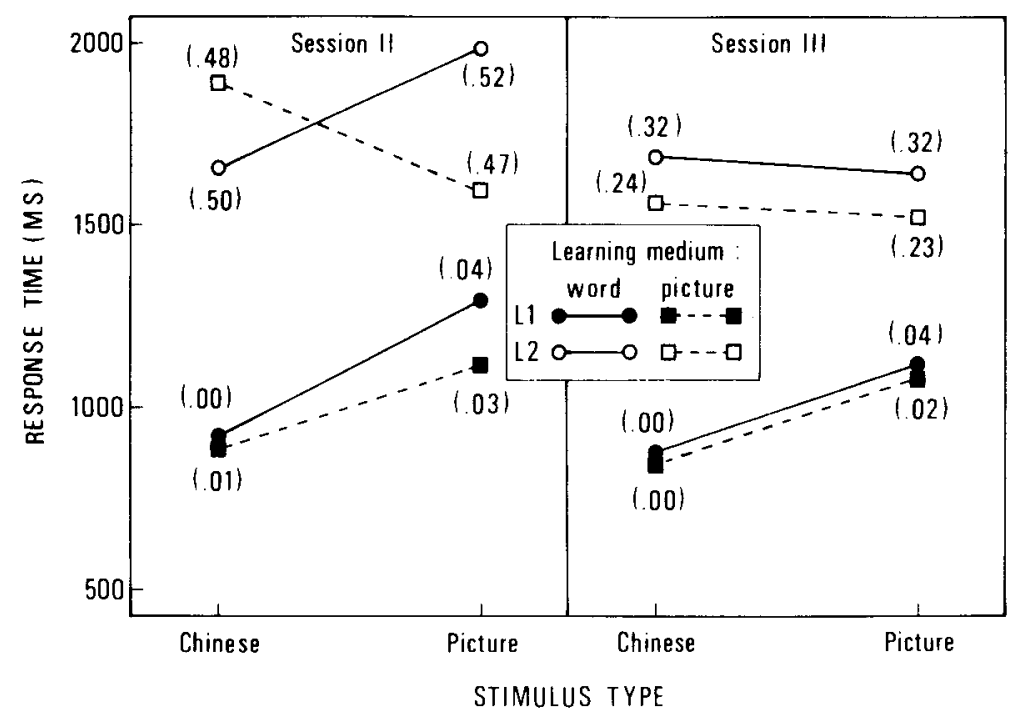

Figure 4. Mean response times in naming or translating Chinese words (L1) and pictured objects as a function of subject group and session in Experiment 3 (error rates in parentheses). 
cant difference was found between the two sessions when the response language was in Chinese $(M s=2 \%$ and $1.5 \%)$. This result clearly suggests that the subjects were more proficient in Session 3 than in Session 2 in using the newly acquired French words. In addition, as expected, more errors were made when the response language was French rather than Chinese in both Sessions 2 and $3[t \mathrm{~s}(38)>8.9, p s<.01]$. These patterns of results are consistent with those of the response time data. There were no indications of a speed-accuracy tradeoff.

\section{GENERAL DISCUSSION}

The purpose in the present set of experiments was to determine whether level of proficiency in a non-native language and learning strategy could affect patterns of lexical processing in the new language. The results of Experiment 1 showed that the subjects' pattern of lexical processing changed systematically with their proficiency in the new language: More proficient subjects were equally efficient in translating $\mathrm{L} 1$ into $\mathrm{L} 2$ and in picture naming in $\mathrm{L} 2$, whereas beginning subjects were more efficient in naming than in translating. Experiment 2, with more homogeneous subjects than those in Experiment 1, demonstrated that college subjects were more efficient in translating than in picture naming in French at the beginning stage of learning a new language. However, as the subjects received more training in the study phase, despite the fact that they only used L1 words to acquire new words, they could respond equally efficiently to both the training stimulus and the other stimulus type. In Experiment 3, two media ( $\mathrm{L} 1$ words or pictures) were adopted for teaching the subjects words in a novel language, and the subjects responded more efficiently to the kind of stimulus used in the study trials at the beginning stage of learning. These findings thus provide evidence for the suggestion of Chen and Leung (1989) that both level of proficiency in a non-native language and strategy of learning the new language are important determinants for the pattern of processing translation equivalents in the native and non-native languages.

The present results with child beginners in a new language stand in interesting contrast with findings of previous research with adult beginners (e.g., Chen \& Leung, 1989; Kroll \& Borning, 1987; Kroll \& Curley, 1987), but they are consistent with other findings with child beginners (e.g., Chen \& Leung, 1989). Chen and Leung, for example, demonstrated that adult beginners use L1 words, but not pictorial representations as did child beginners in the present study, to mediate L2 words. Although the child beginners in the present study differed in a number of ways from the adult beginners, two differences may be crucial: level of proficiency in L1 and learning strategy (Chen \& Leung, 1989). Adult beginners usually have good command of their first language, but this is not necessarily the case with child beginners. Thus, adult beginners can easily use $\mathrm{L} 1$ words to acquire words in a new language, whereas child beginners may rely on concrete learning media such as pictures for such an end. In fact, the results of Experiment 3 confirmed the idea that learning strategy is a main determinant in lexical processing in a non-native language (see, e.g., Galloway, 1982 , and Kiyak, 1982, for a similar suggestion and relevant results).

Note that Experiments 2 and 3 in the present study were direct attempts to vary experimentally both the conditions under which words in a new language are acquired and the amount of experience with the language. Generally, these factors have not been experimentally induced in other studies of bilingual lexical processing (e.g., Chen \& Leung, 1989; Kiyak, 1982; Kroll \& Borning, 1987; Kroll \& Curley, 1987; Potter et al., 1984). Despite this difference, however, the present results are generally in line with those found in the other studies and are consistent with the different models shown in Figure 1. The only exception was that the subjects in Experiments 2 and 3 could respond equally efficiently to both translating and naming tasks, indicating that the subjects were probably using amodal concepts to mediate the acquired L2 words, after about $30 \mathrm{~min}$ of learning an unfamiliar language, but a similar pattern of response was not achieved with more than 2 years of classroom learning of a new language (see, e.g., Chen \& Leung, 1989; Kroll \& Borning, 1987; Kroll \& Curley, 1987; but cf. Potter et al., 1984).

It is not clear why it is easier and faster to achieve a concept-mediation pattern of response (i.e., subjects respond equally efficiently to both translating and naming in L2 tasks) for acquiring a new language in an experimental setting than in a natural setting. This may be due to the fact that subjects in the experimental setting only have to learn a small set of words, whereas learners of a new language in a natural situation need to learn a much larger set of material. In addition, it is possible that recency of exposure is also at work, since the learning phase was followed almost immediately by the test phase in the Experiments 2 and 3. This, however, is not necessarily the case in a natural learning situation. These are issues that warrant future research.

In short, the present results are clearly inconsistent with the hypothesis that beginning and proficient users of a nonnative language use similar strategies for processing words in their new language. Rather, the outcome is exactly what is to be expected on the basis of the intermediate hypothesis (Chen \& Leung, 1989). The present findings, in conjunction with results of relevant studies (e.g., Chen \& Leung, 1989; Kroll \& Borning, 1987; Kroll \& Curley, 1987), thus suggest that proficiency in a non-native language and strategy of learning the new language are two main determinants for the patterns of lexical processing in bilinguals.

\section{REFERENCES}

BAJo, M.-T. (1988). Semantic facilitation with pictures and words. Journal of Experimental Psychology: Learning, Memory, \& Cognition, 14, 579-589.

Biederman, I., \& Tsao, Y. C. (1979). On processing Chinese ideo- 
graphs and English words: Some implications from Stroop-test results. Cognitive Psychology, 11, 125-132.

CHEN, H.-C., \& Ho, C. (1986). Development of Stroop interference in Chinese-English bilinguals. Joumal of Experimental Psychology: Learning, Memory, \& Cognition, 12, 397-401.

Chen, H.-C., \& Leung, Y. S. (1989). Patterns of lexical processing in a non-native language. Journal of Experimental Psychology: Learning, Memory, \& Cognition, 15, 316-325.

CHEN, H.-C., \& NG, M.-L. (1989). Semantic facilitation and translation priming effects in Chinese-English bilinguals. Memory \& Cognition, 17, 454-462.

Galloway, L. M. (1982). Bilingualism: Neuropsychological consideration. Journal of Research \& Development in Education, 15, 12-28.

JIN, Y.-S., \& FisCHLER, I. (1987, March). Effects of concreteness on cross-language priming of lexical decision. Paper presented at the meeting of the Southeastern Psychological Association, Atlanta, GA.

KirSner, K., Smith, M. C., Lockhart, R. S., King, M. L., \& JAIN, M. (1984). The bilingual lexicon: Language-specific units in an integrated network. Journal of Verbal Learning \& Verbal Behavior, 23, 519-539.

KIYAK, H. A. (1982). Interlingual interference in naming color words. Journal of Cross-Cultural Psychology, 13, 125-135.

Kroll, J. F., \& BorNING, L. (1987, November). Shifting language representations in novice bilinguals: Evidence from sentence priming. Paper presented at the annual meeting of the Psychonomic Society, Seattle, WA.
Kroll, J. F., \& Curley, J. (1987). Lexical memory in novice bilinguals: The role of concepts in retrieving second language words. In M. M. Gruneberg, P. E. Morris, \& R. N. Sykes (Eds.), Practical aspects of memory: Current research and issues (Vol. 2, pp. 389-395). London: Wiley.

Potter, M. C., FAulconer, B. A. (1975). Time to understand pictures and words. Nature, 253, 437-438.

Potter, M. C., Kroll, J. F., Yachzel, B., Carpenter, E., \& SherMAN, J. (1986). Pictures in sentences: Understanding without words. Journal of Experimental Psychology: General, 115, 281-294.

Potter, M. C., So, K.-F., Von Eckardt, B., \& Feldman, L. B. (1984). Lexical and conceptual representation in beginning and proficient bilinguals. Journal of Verbal Leaming \& Verbal Behavior, 23, 23-38.

SChWANENFlugel, P. J., \& ReY, M. (1986). Interlingual semantic facilitation: Evidence for a common representational system in the bilingual lexicon. Journal of Memory \& Language, 25, 605-618.

SNodgrass, J. G. (1984). Concepts and their surface representations. Journal of Verbal Learning \& Verbal Behavior, 23, 3-22.

VANDERWART, M. (1984). Priming by pictures in lexical decision. Journal of Verbal Learning \& Verbal Behavior, 23, 67-83.

(Manuscript received April 4, 1989; revision accepted for publication September 12, 1989.) 